\title{
Leveraging Protocol Knowledge in Slack Matching
}

\author{
Girish Venkataramani \\ Carnegie Mellon University \\ 5000 Forbes Ave \\ Pittsburgh, PA 15213 \\ girishv@ece.cmu.edu
}

\author{
Seth C. Goldstein \\ Carnegie Mellon University \\ 5000 Forbes Ave \\ Pittsburgh, PA 15213 \\ seth@cs.cmu.edu
}

\begin{abstract}
Stalls, due to mis-matches in communication rates, are a major performance obstacle in pipelined circuits. If the rate of data production is faster than the rate of consumption, the resulting design performs slower than when the communication rate is matched. This can be remedied by inserting pipeline buffers (to temporarily hold data), allowing the producer to proceed if the consumer is not ready to accept data. The problem of deciding which channels need these buffers (and how many) for an arbitrary communication profile is called the slack matching problem; the optimal solution to this problem has been shown to be NP-complete.

In this paper, we present a heuristic that uses knowledge of the communication protocol to explicitly model these bottlenecks, and an iterative algorithm to progressively remove these bottlenecks by inserting buffers. We apply this algorithm to asynchronous circuits, and show that it naturally handles large designs with arbitrarily cyclic and acyclic topologies, which exhibit various types of control choice. The heuristic is efficient, achieving linear time complexity in practice, and produces solutions that (a) achieve up to $60 \%$ performance speedup on large media processing kernels, and (b) can either be verified to be optimal, or the approximation margin can be bounded.
\end{abstract}

\section{INTRODUCTION}

A pipelined circuit is well-balanced if all computation stages are allowed to execute as soon as the input data is available. Stalls in the pipeline occur due to various reasons; one fundamental cause of pipeline stalls is a mis-match between the data production and consumption rates. Consider the example in Fig. 1a. It shows two pipelined circuit loops, $\mathrm{C} 1$ and $\mathrm{C} 2$, executing concurrently, which must synchronize twice; at stages $\mathrm{X}$ and $\mathrm{Y}$. Assuming that the delay along cycle $\mathrm{C} 1$ is much greater than the delay along $\mathrm{C} 2$, the critical cycle of execution for this circuit is shown in Fig. 1b. This critical cycle passes not just through forward edges of the graph, but also through a backward edge (along channel AX). Such backward edges are used to acknowledge previously produced data items. Stage A cannot generate new data until such an acknowledgment is received from X.

This bottleneck occurs because of a re-convergent path that is unbalanced, $\mathrm{Y}-\mathrm{X}>(\mathrm{Y}-\mathrm{A}+\mathrm{A}-\mathrm{X})$. Data items from previous iterations clog up the shorter path, thus constricting the progress of A until $\mathrm{X}$ consumes the waiting data items. Inserting the buffer acts as temporary storage for new data items, thus enabling A to continue along path AY; the result is a shorter critical cycle latency. In this paper, we

Permission to make digital or hard copies of all or part of this work for personal or classroom use is granted without fee provided that copies are not made or distributed for profit or commercial advantage and that copies bear this notice and the full citation on the first page. To copy otherwise, to republish, to post on servers or to redistribute to lists, requires prior specific permission and/or a fee.

ICCAD '06 November 5-9, 2006, San Jose, CA

Copyright 2006 ACM 1-59593-389-1/06/0011 ...\$5.00.

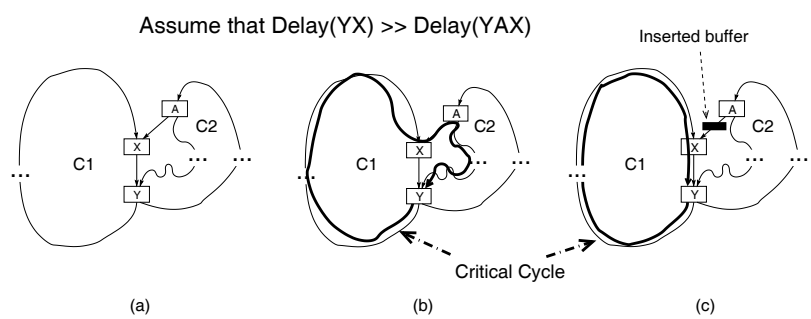

Figure 1: (a) An example demonstrating the need for slack matching; (b) the critical cycle before, and (c) after slack matching.

show that backward edges along the critical cycles are indicative of the presence of bottlenecks. The backward edge is manifest in different forms and patterns depending on the design style, e.g., in asynchronous [9] and latency insensitive synchronous systems [6], it is an explicit control signal representing the acknowledgment of a handshake; for a synchronous FIFO, it may be the fifo_full signal. When the critical cycle of execution is free of such backward edges, then it represents the largest cycle in the circuit constructed from just forward edges. This cycle is referred to as the algorithmic cycle of the circuit, and has been shown to be a performance bound for a given pipelined circuit design [1,3], and is free of communication bottlenecks.

Slack matching is an optimization that determines the minimum number of buffers that must be inserted in order to guarantee that the critical cycle of execution is the algorithmic cycle. Previous approaches to slack matching $[1,8,6]$ have cast the optimization as a constraint satisfaction problem that meets the algorithmic cycle time constraint. The complexity of these solutions are NP-complete. Our solution is an efficient heuristic which is inspired by examining the properties of the critical cycle, which we refer to as the Global Critical Path (GCP) [12]. We observe that: (a) it is possible to accurately model the GCP for a circuit in a given initial state; (b) the backward edges that represent communication bottlenecks will manifest themselves as a precise pattern on the GCP, whose topology is directly correlated to the protocol used to communicate over a given channel; and (c) when a buffer is inserted on such a channel, it is possible to eliminate the bottleneck, and to efficiently and accurately re-compute the GCP to reflect the new critical cycle.

Based on these observations, we propose an algorithm that starts with the GCP for an initial state of the circuit, examines it for bottleneck patterns representing mis-matched communication rates, inserts pipeline buffers to eliminate the bottlenecks, updates the GCP and looks for more opportunities. This is repeated until no more bottlenecks can be removed from the GCP. We show that this algorithm is efficient and, in practice, achieves linear time complexity in the number of communication channels in the system. The algorithm is a heuristic since we cannot prove that the algorithmic cycle time will be met, or that the minimum number of buffers will be inserted. However, experimental results indicate that the heuristic achieves a solution that is provably either optimal, or is within a 5\% approxi- 

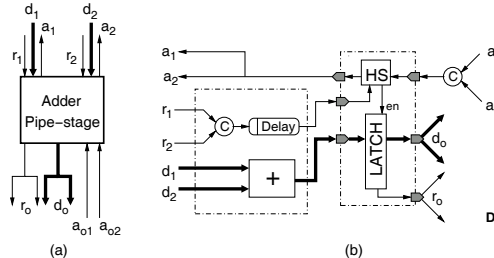

(b)

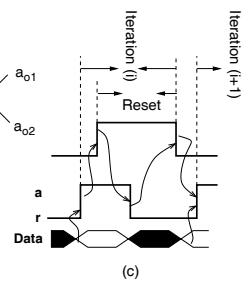

Figure 2: (a) An asynchronous communication channel is associated with two control signals (req and ack), (b) a typical asynchronous pipeline stage, and (c) A 4-phase handshake protocol.

mation margin. The result is significant performance improvement (up to $60 \%$ speedup) and improved energy efficiency, as reported by post-layout simulations.

In this paper, we focus on an asynchronous communication protocol, the four-phase bundled data protocol [4]. However, the concepts proposed can be adapted to other protocols as well. Section 2 introduces the modeling and analysis framework that infers the necessary metrics for use in the optimization phase. Section 3 describes the slack matching algorithm, which we compare to previous work in Section 4. In Section 5, we report experimental results, and conclude in Section 6.

\section{MODELING AND ANALYSIS}

Asynchronous circuit design is characterized by the absence of a global synchronization signal, e.g., a clock. Fig. 2a shows the interface of a typical asynchronous stage. Each data channel carries additional control signals, which participate in the handshake protocol. Fig. 2b shows the architecture of this stage: it has a controller, $H S$ unit, which implements the local handshaking and latches the result. Fig. 2c shows a four-phase handshake protocol [4], which is commonly used in asynchronous designs for data transfers between stages; we apply our slack matching algorithm in the context of this protocol.

\subsection{System Modeling}

We model the performance behavior of these circuits at the pipeline stage granularity. Specifically, we capture dependence relations between the handshake events at the pipeline stage interface. An analysis phase then computes the steady-state timing dependencies between these events, which form the input to the slack matching algorithm. We formally describe this modeling and analysis phase in the remainder of this section.

The behavior of a given circuit is captured by the system, $N=$ $\left(E, B\right.$, In, Out $\left., X, R, M, M_{0}\right)$. An event, $e \in E$, represents a transition on a control signal in the circuit. It is alive when the equivalent circuit transition has fired. The set of all live events is given by $M$, and $M_{0}$ is the set of events initially live. A behavior, $b \in B$, defines how an event becomes alive. It is associated with some input events $\left(\operatorname{In}: B \mapsto 2^{E}\right)$, and generates some output events (Out $\left.: B \mapsto 2^{E}\right)$. It is satisfied when all its input events are alive $(\operatorname{In}(b) \subseteq M)$. In the absence of choice [13], a behavior can fire once it is satisfied, and this will make all its outputs live.

Choice and conditional flow semantics [13] are modeled by allowing multiple behaviors to fire the same events. Thus, $\exists b_{1}, b_{2} \in B$, s.t., $\operatorname{Out}\left(b_{1}\right) \cap \operatorname{Out}\left(b_{2}\right) \neq \emptyset$. For unique (deterministic) choice, only one of these behaviors can be satisfied at any instant, i.e., $\operatorname{In}\left(b_{1}\right) \cup \operatorname{In}\left(b_{2}\right) \not \subset$ $M$ is an invariant. Arbitration (non-deterministic) choice is modeled by specifying mutually exclusive behaviors, $X: B \mapsto 2^{B}$. A member $(X(b) \subseteq B)$ is a set of behaviors which may be satisfied simultaneously, but their outputs are mutually exclusive. Thus, $b$ fires iff: $\forall b^{\prime} \in X(b)$, Out $\left(b^{\prime}\right) \cap M=\emptyset$. If two members of $X(b)$ are satisfied in the same instant, then one of them is probabilistically chosen to fire, reflecting the non-deterministic firing semantics of arbitration behaviors. If $b$ is a non-choice or unique choice behavior, $X(b)=\emptyset$.
Finally, we define $R: E \mapsto E$, which specifies how an event becomes dead; an event $e \in M$ is removed from $M$ when $R(e)$ becomes alive. For example, the rising transition of a circuit signal will render the falling transition of the same signal as dead. The following models the adder in Fig. 2a, assuming that it implements the protocol described in Fig. 2c:

$$
\begin{array}{lll}
E= & \left\{r_{1} \uparrow, r_{1} \downarrow, r_{2} \uparrow, r_{2} \downarrow, a_{1} \uparrow, a_{1} \downarrow, a_{2} \uparrow, a_{2} \downarrow,\right. \\
& \left.r_{o} \uparrow, r_{1} \downarrow, a_{1} \uparrow, a_{o_{1}} \downarrow, a_{o_{2}} \uparrow, a_{o_{2}} \downarrow\right\} \\
B= & \left\{b_{1}, b_{2}, b_{3}\right\} & X=\emptyset \\
\operatorname{In}\left(b_{1}\right)= & \left\{r_{1} \uparrow, r_{2} \uparrow, a_{o_{1}} \downarrow, a_{o_{2}} \downarrow\right\}, & \text { Out }\left(b_{1}\right)=\left\{r_{o} \uparrow, a_{1} \uparrow, a_{2} \uparrow\right\} \\
\operatorname{In}\left(b_{2}\right)= & \left\{a_{o_{0}} \uparrow, a_{o_{2}} \uparrow\right\}, & \text { Out }\left(b_{2}\right)=\left\{r_{o} \downarrow\right\} \\
\operatorname{In}\left(b_{3}\right)= & \left\{r_{1} \downarrow, r_{2} \downarrow\right\}, & \text { Out }\left(b_{3}\right)=\left\{a_{1} \downarrow, a_{2} \downarrow\right\} \\
M_{0}= & \{e \downarrow \mid e \in E\} & \forall e \in E, R(e \uparrow)=e \downarrow, R(e \downarrow)=e \uparrow
\end{array}
$$

Behavior $b_{1}$ describes how the adder functions. Once its input data channels are valid (indicated by $r_{1} \uparrow$ and $r_{2} \uparrow$ ), and its previous output has been consumed (i.e., $a_{O_{1}} \downarrow$ and $a_{O_{2}} \downarrow$ ), the adder processes its inputs, generates a new output $\left(r_{o} \uparrow\right)$, and acknowledges its inputs $\left(a_{1} \uparrow\right.$ and $\left.a_{2} \uparrow\right)$. Behaviors $b_{2}$ and $b_{3}$ describe the reset phase of the handshake as shown in Fig. 2c. Notice that the sets In and Out only specify the input and output handshake signals of the pipeline stage interface. Thus, behaviors in the model are restricted to control events (handshake events, in particular), implying that internal events, implementation details, and datapath logic are abstracted away. This not only leads to a large reduction in the model size, but also decouples system modeling from system implementation.

\subsection{Model Analysis}

Performance can be analyzed by associating a delay, $D: B \mapsto \mathbb{R}$, with every behavior in the model. This is the expected execution latency through the micro-architectural blocks that $b$ describes. Depending on the system and the environment, these delays may be deterministic or stochastic. The model can now be analyzed by simulating the firing sequence of events and behaviors - when $b \in B$ is satisfied, it fires after $D(b)$ time units, generating its output events, which in turn satisfy other behaviors. This analysis is similar to techniques proposed for analyzing event-rule systems [3], marked graphs [7], and Petri-Nets [13]. The weaknesses of all these techniques are (a) their inability to scale when analyzing large problem sizes (on the order of thousands of events and beyond), and (b) their inability in handling systems with choice.

To overcome these difficulties, we employ a trace-based simulation technique to focus the analysis on the most commonly expected input vectors. Since there is a one-to-one correspondence between events and signal transitions, and between behaviors in the model and microarchitectural blocks in the circuit, we simulate the synthesized and laid out circuit using commercial simulators (like Modelsim), and analyze the model during circuit simulation. This results in an accurate delay model, and our experiments reveal a low overhead (about 6\%) in analyzing the model during simulation.

Slack. The analysis produces slack relations for every $b \in B$; if in the $k^{\text {th }}$ firing, inputs of $b$ (where $|\operatorname{In}(b)|=m$ ) arrived at times, $\left\{t_{1} \leq\right.$ $\left.\cdots \leq t_{i} \leq \cdots \leq t_{m}\right\}$, then slack on event, $e_{i}$ is given by $\operatorname{Slack}^{k}\left(e_{i}, b\right)=$ $\left(t_{m}-t_{i}\right)$. This implies that $e_{i}$ arrived $\operatorname{Slack}^{k}\left(e_{i}, b\right)$ time units before the last arriving event, $e_{m}$, which satisfied $b$. Event, $e_{m}$, with the least slack is referred to as the locally critical event, Crit (b).

Global Critical Path (GCP). If $b_{\text {last }}$ is the last behavior to fire in the timed execution, then we define the GCP as the sequence of behaviors, $\left\langle b_{1}, \ldots, b_{i}, \ldots, b_{\text {last }}\right\rangle$. For any two consecutive $\left(b_{i}, b_{i+1}\right)$ in the GCP, $\operatorname{Crit}\left(b_{i+1}\right) \in \operatorname{Out}\left(b_{i}\right)$. Equivalently, it is also the event sequence, $<$ $e_{1}, \ldots, e_{\text {last }}>$, s.t., $e_{i}=\operatorname{Crit}\left(b_{i}\right)$.

The slack and GCP metrics thus constructed are described for a timed execution [13]. Thus, there may be multiple instances of a given $b$ in the GCP. Since the number of times a behavior fires can be potentially large, we summarize slack and GCP on an untimed model. For a given $b$ that fires $N_{b}$ times, slack on $e_{i} \in \operatorname{In}(b)$ is given by $\operatorname{Slack}\left(e_{i}, b\right)=\left(\sum_{j=1}^{N_{b}} \operatorname{Slack}^{j}\left(e_{i}, b\right)\right) / N_{b}$. Summarizing GCP and slack 


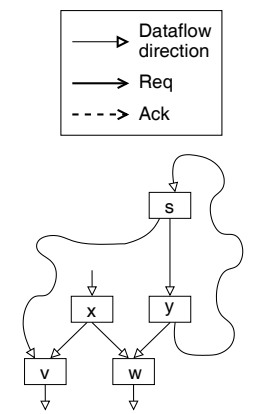

(a)

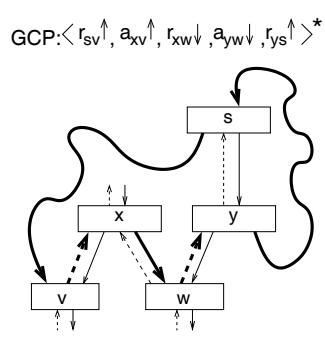

(b)

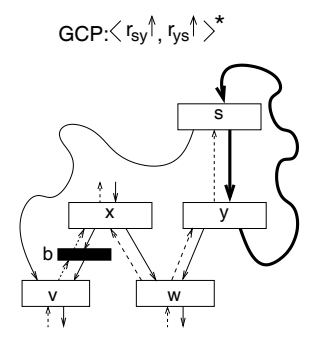

(c)

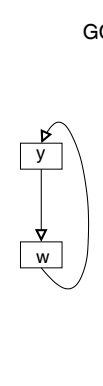

(d)

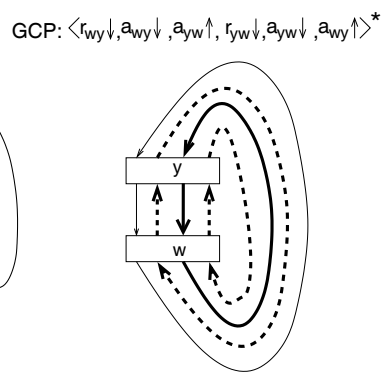

(e)
$\mathrm{GCP}:\left\langle\mathrm{r}_{\mathrm{yb}} \uparrow, \mathrm{r}_{\mathrm{bw}} \uparrow, \mathrm{r}_{\mathrm{wy}} \uparrow\right\rangle^{\star}$

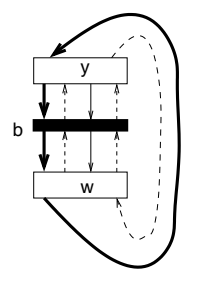

(f)

Figure 3: Two causes of bottlenecks: re-convergent paths (a-c) and short loops (d-f). For each, the figure shows system topology with data dependencies (a,d), the GCP in the presence of a bottleneck (b,d), and the GCP after buffer insertion (c,f). Bold edges mark the GCP.

in this manner leads to information loss, but it also biases the analysis for the common case. These metrics are key components in our slack matching algorithm, which is described in the following section.

\section{SLACK MATCHING}

Beerel [1] notes that there are only two causes for communication bottlenecks: re-convergent paths and short loops. For example, the design in Fig. 1 has a re-convergent path that causes a bottleneck. The key insight to finding the bottlenecks in both these cases is the observation that these bottlenecks are manifest as a precisely defined pattern (critical event sequence) on the GCP. The shape of this sequence is defined by the communication protocol employed.

Re-convergent paths. These are the most common source of bottlenecks in large concurrent systems. To find and eliminate these bottlenecks, an analysis of the communication protocol is essential. In some cases, the re-convergent path is not immediately apparent, but can be uncovered only by examining the protocol interactions. For example, there are apparently no re-convergent paths in Fig. 3a, but when the handshake event interaction is observed, we notice that a reconvergent path exists, forking at stage $s$. The join point, however, is not a single stage, but three stages together $(v, x$ and $w$ ). To understand why, consider Fig. $3 \mathrm{~b}$ which shows for each channel, the Req/Ack signals that implement the 4-phase handshake described in Fig. 2c. The model describing the firing behavior for these signals is similar to the adder example in Section 2.1. Assume that the path $s v$ is much longer than $s y$. If $r_{m n}$ and $a_{m n}$ are the Req/Ack signals of the channel from $m$ to $n$, then $r_{s y} \uparrow$ arrives much earlier than $r_{s v} \uparrow$. Stage $y$ can begin the handshake on channels $y s$ and $y w$, firing $r_{y s} \uparrow$ and $r_{y w} \uparrow$. After this, $w$ acknowledges with $a_{y w} \uparrow$, and the reset phase begins when $y$ generates $r_{y w} \downarrow$. However, the reset phase cannot complete since $w$ must wait for $r_{x w} \downarrow$ from $x$, before generating $a_{y w} \downarrow$. Event $r_{x w} \downarrow$, in turn, is awaiting $a_{x v} \uparrow$, which in turn is awaiting $r_{s v} \uparrow$, the latter representing data along the longer path from $s$. Thus, stages $v, x$ and $w$ are all involved in the handshake along both paths, $s w$ and $s v$, and together they form the join point of the re-convergent path from $s$.

If the cycle $s-y-s$ is shorter than the path $s v$, then, in steady state, $r_{s y} \uparrow$ always arrives earlier than (and is waiting for) $a_{y w} \downarrow$, i.e., the next iteration is throttled due to the late completion of the handshake reset phase of the previous iteration, resulting in a bottleneck; the GCP is: $\left\langle r_{s v} \uparrow, a_{x v} \uparrow, r_{x w} \downarrow, a_{y w} \downarrow, r_{y s} \uparrow\right\rangle^{*}$. In other words, if no bottleneck exists, then a stage should process its inputs as soon as they all arrive. In essence, the appearence of the reset event sequence, $\left\langle a_{x v} \uparrow, r_{x w} \downarrow, a_{y w} \downarrow\right\rangle$ on the GCP is indicative of a slow consumer, resulting in a bottleneck.

If a buffer is inserted on channel $x v$ (Fig. 3c), then the late ack, $a_{x v} \uparrow$ can now be generated early since it no longer needs to wait for $r_{s v} \uparrow$; in other words, the buffer acts as temporary storage for the early arriving input at stage $v$. The ack event can now be generated as soon as $r_{x v} \uparrow$ fires, implying that inserting a buffer accelerates the generation of $a_{x v} \uparrow$ by the amount of time $r_{x v} \uparrow$ was kept waiting, which is noth-

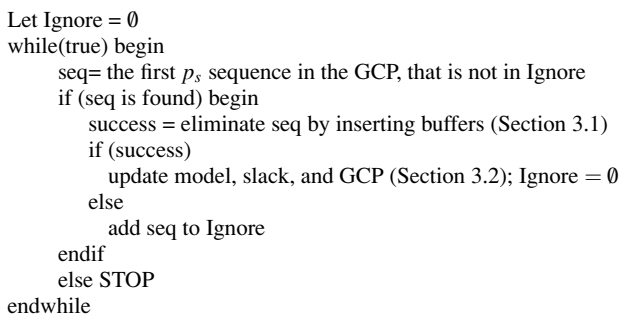

Figure 4: The overall slack matching algorithm.

ing but $\operatorname{Slack}\left(r_{x v} \uparrow, b^{\prime}\right)$, where $b^{\prime}$ fires $a_{x v} \uparrow$. This is a key insight in the bottleneck elimination algorithm as described in Section 3.1. The early ack event, $a_{x v} \uparrow$, in turn, produces early $r_{x w} \downarrow$ and $a_{y w} \downarrow$ events, thus eliminating the bottleneck. The GCP of the design now shifts to the topology shown in Fig. 3c.

Short Loops. A similar situation occurs in the presence of short loops as shown in Fig. 3d-f. In this case, the forward delay to transmit data around the loop (i.e., along path $\left[r_{y w} \uparrow \rightarrow r_{w y} \uparrow\right]$ ) is shorter than the delay to reset the handshake backwards along the same loop, resulting in the GCP shown in Fig. 3e. Again, the insertion of a buffer hastens the generation of the critical events resulting in a GCP devoid of the handshake reset events. Notice that after buffer insertion, the GCP is shorter in both cases (Fig. 3c,f).

In summary, we note that for any communication protocol, we can identify two event sequences $-p_{d}$ signifies the generation of new data by the producer, and $p_{s}$ is a synchronization path, which signifies the acceptance of this data by the consumer. For example, in the four phase protocol, $p_{d}=\langle r \uparrow\rangle$ and $p_{s}=\langle a \uparrow, r \downarrow, a \downarrow\rangle$; for a synchronous FIFO with protocol signals, $\{$ enq, deq, full,empty $\}, p_{d}=$ $\langle e n q, \overline{e m p t y}\rangle$, and $p_{s}=\langle d e q, \overline{f u l l}\rangle$. Ideally, we would like $p_{s}$ to occur in parallel, before the next iteration's data shows up. If not, we will have new data, which we are not ready to process, implying a bottleneck. It can be shown that for any protocol, the GCP can be captured by the regular expresion: $\left\langle p_{d}^{*}, p_{s}^{*}\right\rangle^{*}$. In fact, the short-loop bottlenecks will always feature at least two consecutive $p_{s}$ sequences on the GCP, or as in the pathological example in Fig. 3e, the GCP may be just $\left\langle p_{s}\right\rangle^{*}$. The GCP for a bottleneck-free system, however, will be $\left\langle p_{d}\right\rangle^{*}$.

Fig. 4 describes the overall algorithm for eliminating $p_{s}$ sequences from the GCP. It iteratively finds a $p_{s}$ sequence, inserts buffers and updates the system model. The $p_{s}$ sequences are considered for elimination in topological-sort order of the GCP. This is because it is highly likely that such sequences are the cause for the appearance of others downstream. The remainder of this section describes how we eliminate $p_{s}$ sequences from the GCP, and how the GCP and system model are updated once a buffer is inserted. We end the section with an analysis of the complexity and optimality of the algorithm. Although the algorithm is described for a 4-phase asynchronous handshake proto- 


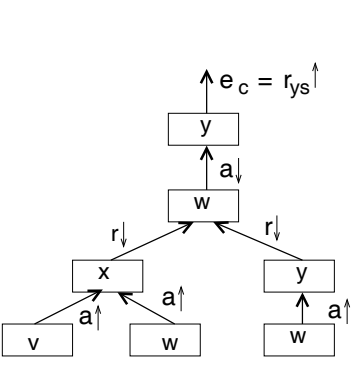

(a)

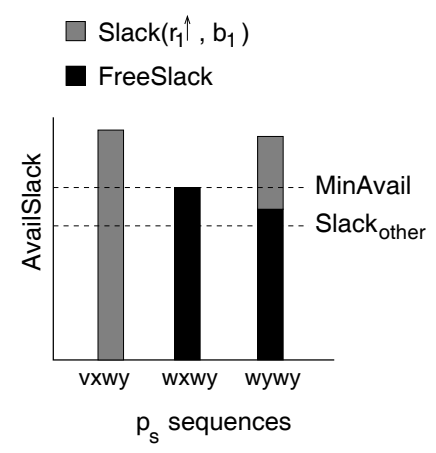

(b)
Figure 5: A visual of (a) all the $p_{s}$ paths that can precede $r_{y s}$ in Fig. $3 b$ (ignoring the $p_{s}$ paths along ys for simplicity), and (b) computation of various slack values.

col, it can be extended for other protocols by appropriately defining the model and the $p_{s}$ and $p_{d}$ event sequences.

\subsection{Bottleneck Elimination}

Observe that the $p_{s}$ sequence $\left\langle a_{x v} \uparrow, r_{x w} \downarrow, a_{y w} \downarrow\right\rangle$ is on the GCP in Fig. 3b, because $r_{s y} \uparrow$ arrives earlier at the behavior, call it $b_{c}$, which generates $r_{y s} \uparrow$. To make the $p_{s}$ sequence non-critical, we must generate it sooner by at least $\operatorname{Slack}\left(r_{s y} \uparrow, b_{c}\right)$, which is the slack on the waiting event. In general, let $e_{c}$ (fired by $b_{c}$ ) be the event that succeeds the $p_{s}$ sequence on the GCP, and Slack ${ }_{\text {other }}=\operatorname{Slack}\left(e_{c}, b_{c}\right)$ be the slack on the waiting event at $b_{c}$ that $p_{s}$ must offset.

Since the $p_{s}$ sequence on the GCP is a chain of locally critical events, firing the first event of $p_{s}$ (call it $e_{1}$ generated by behavior $b_{1}$ ) earlier, will accelerate the firing of all events in $p_{s}$. This may be achieved by inserting a buffer along the channel, $\operatorname{chan}\left(e_{1}\right)$, associated with $e_{1}$; a necessary condition for this, however, is that the $p_{d}$ event of chan $\left(e_{1}\right)$, which is an input to $b_{1}$ must have positive, non-zero slack. For example, in Fig. 3b, $e_{1}$ is $a_{x v} \uparrow$; inserting a buffer along $x v$ can hasten the generation of $a_{x v} \uparrow$ only if $r_{x v} \uparrow$ (which is the $p_{d}$ event of $x v)$ has positive, non-zero slack. Inserting a buffer ensures that the locally critical event which was constricting $p_{s}$ would not affect its firing anymore, e.g., $r_{s v} \uparrow$ is not necessary to fire $a_{x b} \uparrow$ in Fig. 3c. Fur-

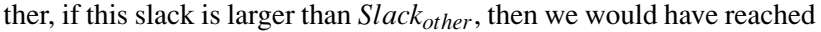
our goal. Thus, to yield a GCP free of $p_{s}$ sequences, we must ensure that this condition can be achieved along every $p_{s}$ sequence that could potentially precede $e_{c}$, and appear on the GCP.

Let the set $P_{c}$ represent every $p_{s}$ sequence that could precede a given $e_{c}$ on the GCP. We can visualize this set as a tree, whose root is $e_{c}$, and every path from leaf to root represents a $p_{s}$ sequence in $P_{c}$. The depth of the tree is $\left(\left|p_{s}\right|+1\right)$, which is four in our protocol. For example, Fig. 5a shows this tree for $e_{c}=r_{y s} \uparrow$ in Fig. 3b. To determine if there is sufficient slack to offset Slack ${ }_{\text {other }}$ at $b_{c}$ (which fires $e_{c}$ ), we compute the following:

$$
\begin{array}{ll}
\text { FreeSlack }\left(p_{s}\right)= & \text { Slack }\left(e_{\left|p_{s}\right|}, b_{c}\right)+\sum_{i=2}^{\left|p_{s}\right|} \operatorname{Slack}\left(e_{i-1}, b_{i}\right) \\
\operatorname{AvailSlack}\left(p_{s}\right)= & \text { FreeSlack }\left(p_{s}\right)+\operatorname{Slack}\left(r_{1} \uparrow, b_{1}\right) \\
\text { MinAvail }= & \text { MIN }\left(\operatorname{AvailSlack}\left(p_{s}\right)\right), \forall p_{s} \in P_{c} \\
& >\operatorname{Slack}_{\text {other }} \\
\text { MinAvail } & \left.>\text { FreeSlack }_{p_{s}}\right)
\end{array}
$$

where $e_{i}$ is the $i^{t h}$ event in a given $p_{s}$ sequence (thus $e_{\left|p_{s}\right|}$ is an $a \downarrow$ event, e.g., $a_{y w} \downarrow$ in Fig. 3b), behavior $b_{i}$ fires $e_{i}, r_{1} \uparrow$ is the $p_{d}$ event of chan $\left(e_{1}\right)$, e.g., $r_{x v} \uparrow$ in Fig. $3 \mathrm{~b}$. This computation is illustrated in Fig. 5b. FreeSlack $\left(p_{s}\right)$ specifies, for each path through the tree (see Fig. 5a), the amount slack the $p_{s}$ sequence has since the firing of its first event, $e_{1}$, until the firing of $e_{c}$. There exists at least one $p_{s} \in P_{c}$, i.e., one path through the tree, whose FreeSlack $\left(p_{s}\right)$ value is zero; this is the sequence on the GCP, e.g., vxwy in Fig. 5b.

$\operatorname{AvailSlack}\left(p_{s}\right)$ specifies the slack that would be available if we were to insert a buffer on $\operatorname{chan}\left(e_{1}\right)$, and apportion the slack from the

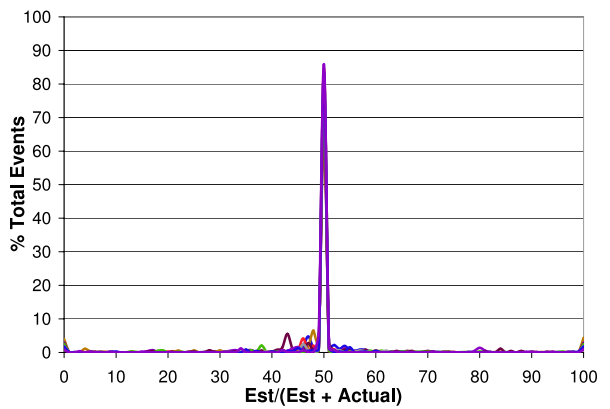

Figure 6: The error distribution of estimating slack. If $S_{e}$ and $S_{r}$ are the estimated and real slack for an event, then the $x$-axis shows $\frac{S_{e}}{S_{e}+S_{r}} \times 100$; for each error value, the graph shows the percentage of total events with this error value.

waiting $r \uparrow$ event of $\operatorname{chan}\left(e_{1}\right)$. This is equivalent to the slack on the $r \uparrow$ event of the channel between a leaf of the tree and its parent, e.g., slack of $r_{x v} \uparrow$. MinAvail, the minimum of all AvailSlack, specifies the maximum slack that can be generated by inserting buffers on the leaves of the tree. If this quantity is larger than Slack ${ }_{\text {other }}$, then some event that is not in any $p_{s} \in P_{c}$ (e.g., $r_{s y} \uparrow$ in Fig. $3 \mathrm{~b}$ ), will become locally critical thus eliminating $p_{s}$ from the GCP. Thus, (1) represents a necessary and sufficient condition for obtaining a GCP free of $p_{s}$ sequences.

For a given $p_{s}$ path in the tree, if FreeSlack $\left(p_{s}\right)$ is already larger

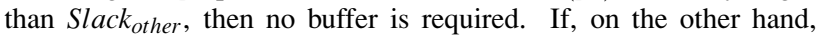
FreeSlack $\left(p_{s}\right)<$ Slack $_{\text {other }} \leq$ AvailSlack $\left(p_{s}\right)$, then we must insert a buffer on the leaf of the path, in order to leverage AvailSlack. We argue that even if FreeSlack $\left(p_{s}\right)<\operatorname{AvailSlack}\left(p_{s}\right)<$ Slack $_{\text {other }}$, we should still insert a buffer; although this buffer will not help eliminate $p_{s}$ from the GCP, it will still help in accelerating its firing by $\left(\operatorname{AvailSlack}\left(p_{s}\right)-\right.$ FreeSlack $\left.\left(p_{s}\right)\right)$, thus shortening the length of the GCP. Both these conditions together are captured by (2), which if satisfied results in a buffer insertion. Being conservative, however, a buffer is inserted only if this difference is larger than the latency through the buffer itself; otherwise, the output, $r \uparrow$, of the buffer will be locally critical downstream, but not necessarily on the GCP.

\subsection{Metric Update}

The above algorithm described how to eliminate one $p_{s}$ pattern from the GCP; there may however be many more, and eliminating one may introduce others. Before looking for more opportunities, however, the model and the slack/GCP metrics must be updated to reflect changes due to the newly inserted buffer. For example, insertion of buffer, $b$, along channel $x v$ in Fig. $3 c$, eliminates an existing channel, $x v$, and introduces two new channels, $x b$ and $b v$, resulting in a corresponding change to the event set of the model. The slack values for the new events can be trivially determined. For example, the slack on the $r_{b v} \uparrow$ is the slack on the event it replaced, $r_{x v} \uparrow$, minus the delay of generating $r_{b v} \uparrow$ from $b$. Changes to slack on the other local events can be similarly determined.

Next, these changes are propagated globally. This is done by computing the minimum slack at each $b \in B$, and if this value has changed, then the difference is propagated to $b^{\prime}$, iff: $\operatorname{Out}(b) \cap \operatorname{In}\left(b^{\prime}\right) \neq \emptyset$. The effect of slack change needs to be propagated only once throughout the model, i.e., the propagation stops if the same input at the same behavior is reached again. This is because the initial slack from the analysis is a steady state property (in fact, an asymptote in systems without choice). A local change to slack implies a change to the steady state value, whose effect needs to be propagated to other events in the system.

Clearly, the slack and GCP updates are heuristics due to the lack of iteration history. We validated our algorithms by comparing the slack estimated by this update technique against a complete re-analysis of the model. The result of the comparison is shown in Fig. 6. If es- 
timated slack is $S_{e}$ and actual slack is $S_{r}$, then the X-axis shows the error value as $E r r=\frac{S_{e}}{S_{e}+S_{r}} \times 100$; i.e., $E r r=50$ indicates an accurate estimation. Each trendline represents a benchmark from Table 1, and the plot shows the percentage of all events with a given error value. These results indicate that, on average, our slack estimation is accurate for more than $75 \%$ of all events, and more than $90 \%$ of events are within a $\pm 10 \%$ error margin, validating this update method. The reasons for the inaccuracies are two-fold: (1) lack of iteration history, and (2) some latency estimates $(D(b))$ may not match those from actual post-layout values.

\subsection{Complexity and Optimality}

The computational complexity of our algorithm (as described in Fig. 4) is summarized as follows: (1) the complexity of the initial trace-based model analysis and slack/GCP construction is $O(|E| k)$, where $k$, the number of iterations simulated, depends on the analyzed design; (2) finding a $p_{s}$ pattern on the GCP has $O(|G C P|)$ time complexity, i.e., $O(|E|)$ in the worse-case; (3) eliminating $p_{s}$ depends on the fan-out and fan-in of the channels in the pattern. If $f_{o}$ and $f_{i}$ are the maximum fan-out and and fan-in for any behavior in the model, then $O\left(f_{o}^{2} f_{i}\right)$ is the worst-case complexity; on average, $f_{o}$ and $f_{i}$ are about two or three; (3) updating slack and GCP each have $O(|E|)$ worst-case complexity, while updating the model occurs in constant time; (4) finally, we must bound the number of iterations the algorithm needs for convergence. In the worst-case (one buffer insertion per iteration): $\sum_{i} \frac{\left(C_{i}-C_{o}\right)}{D_{b}}, \forall C_{i}>C_{o}$, where $C_{o}$ is the largest (algorithmic) cycle composed of only $r \uparrow$ events, and $D_{b}$ is the delay through a pipeline buffer. That is, in the worst-case, we would have to insert buffers on all mismatched cycles. Thus, the number of iterations has $O(|E|)$ time complexity. In practice, as reported in Table 1, the algorithm converges within five iterations in most cases. The overall complexity is $O(|E| k)+O\left(|E|^{2} f_{o}^{2} f_{i}\right)$ in the worst-case, and $O(|E|)$ in practice, i.e., the heuristic scales linearly with the number of handshake communication channels in the model.

Finally, we address the question of optimality. There are two axes of optimality in slack matching: (a) the critical cycle must be the algorithmic cycle time, and (b) the number of pipeline buffers inserted to achieve this constraint is minimized. If the GCP, which is the critical cycle, is made up of only $p_{d}$ events (which are $r \uparrow$ in the 4-phase protocol) and does not contain any newly introduced pipeline buffers, then it is guaranteed to be the algorithmic cycle. Observe, however, that in the case of short loops (Fig. 3f), buffers are always inserted on the GCP; thus the presence of buffers on the GCP does not imply non-optimality, but their absence guarantees optimality. The second optimality property, however, cannot be proven without an exhaustive analysis akin to [1]. While we cannot prove whether or not the heuristic algorithm can find an optimal solution, we can determine an approximation margin, $\varepsilon$, which specifies how far off the optimal a generated solution could be, in the worst case. If the GCP consists of only $p_{d}$ events and does not contain any newly inserted buffers, then $\varepsilon=0$, and the solution is optimal. If the GCP contains $N_{B}$ new buffers, the delay through the buffer for generating $p_{d}$ is $D(b u f f)$, and the algorithmic cycle time constraint is $C_{o}$, then $\varepsilon=\frac{N_{B} \times D(b u f f)}{C_{o}}$, in the worst case. We note that since $N_{B}$ is typically very small (see Table 1$), \varepsilon$ is also very small ( $<5 \%$ in our experiments).

\section{RELATED WORK}

Proposals for slack matching a circuit design can be grouped into two categories: delay insertion to optimize the clock cycle time in synchronous circuits $[2,10]$, and those that minimize the global cycle time in data driven systems, e.g.,Latency Insensitive synchronous (LIS) designs [6] and asynchronous circuit designs [1,8]. All these proposals take a similar approach to the solution: a target timing constraint is defined, and equations for circuit timing dependencies are

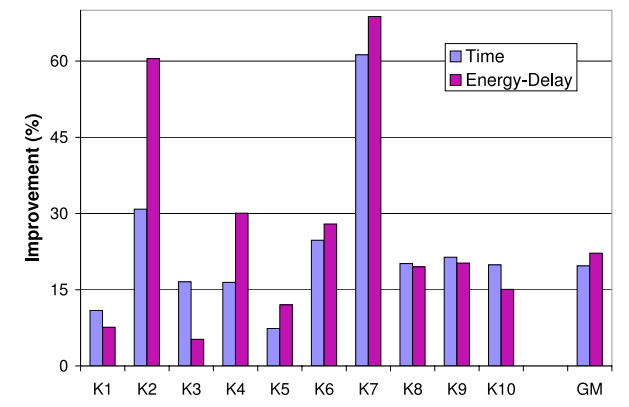

Figure 7: Performance and energy-delay improvements after slack matching. The benchmarks are numbered in order of their listing in Table 1 .

set up to meet the given timing constraint, which are then solved by a linear programming optimization.

The latter category $[1,8,6]$, which attacks a problem similar to the one in this paper, defines a constraint satisfaction problem, where one of the constraints is for the critical cycle latency to be equal to the algorithmic cycle latency; the optimization objective is to minimize the number of buffers inserted to achieve this constraint. In contrast, our algorithm finds and eliminates bottlenecks by explicitly modeling them in the GCP. In terms of complexity, previous algorithms generally involve finding the cycle time constraint (the algorithmic cycle), generating a system of linear constraints, and solving the optimization problem. Determining the cycle time constraint in their approaches, and finding the GCP and slack metrics in ours, has comparable complexities. From here, we diverge from previous work. Determining the linear constraints and solving the optimization problem has been shown to be $O\left(m^{2} n^{2}\right)$ [8] and NP-complete respectively (where $m$ and $n$ are the number of edges and nodes in the graph). We decompose the hard problem into smaller sub-problems that are iteratively solved. The overall complexity is $O\left(m^{2} f_{o}^{2} f_{i}\right)$ in the worst-case, and $O(m)$ in practice. In terms of capabilities, previous work cannot generate the LP constraints in the presence of choice. Our heuristic, on the other hand, naturally adapts to systems with all types of choice. The drawback of our approach is that creating smaller sub-problems prevents us from being able to prove its optimality. However, our results indicate that the solutions for many benchmarks are indeed optimal in the algorithmic cycle time.

\section{EXPERIMENTAL RESULTS}

We have implemented our algorithm in a toolflow that automatically synthesizes asynchronous circuits implementing a four-phase, bundled data protocol [11]. Synthesis uses a standard-cell flow, and targets a $[180 \mathrm{~nm} / 2 \mathrm{~V}]$ technology library. A property of our algorithm is that when it insert buffers, it is guaranteed to improve performance as described in Section 3.1. But, when no opportunities exist (i.e., there are no $p_{s}$ sequences on the GCP), it leaves the design unchanged. In this section, we report on the results of the kernels from the Mediabench suite [5] that could be improved. We randomly picked kernels from these benchmarks, and noticed that more than $50 \%$ of those tested could be improved. Table 1 shows these kernels and their sizes in terms of the number of pipeline stages, behaviors and events.

The last column in Table 1 lists the number of iterations the algorithm took to converge, and the number of pipeline buffers inserted in each iteration. More than one pipeline buffer may be inserted per iteration as described in the slack tree analysis algorithm (see Fig. 5). All the generated solutions result in a GCP consisting of only $p_{d}$ events. For seven of these kernels, the GCP does not contain any new buffers, and thus the solution is optimal $(\varepsilon=0)$. For the remainder $(\mathrm{K} 3, \mathrm{~K} 7$ and $\mathrm{K} 10$ ), we have determined that $\varepsilon<5 \%$, in the worst case.

Our experiments indicate that the number of buffers inserted correlates with what caused the $p_{s}$ bottleneck to appear on the GCP. In re-convergent paths, for which the critical event after the $p_{s}$ sequence 


\begin{tabular}{|l|l|l|r|r|r|r|}
\hline Id & Bench & Kernel & $\begin{array}{r}\text { Pipe } \\
\text { Stages }\end{array}$ & Bhvs & Evts & $\begin{array}{r}\text { Buffs per } \\
\text { iteration }\end{array}$ \\
\hline \hline K1 & adpcm_d & adpcm_decoder & 191 & 761 & 1218 & $(5)$ \\
\hline K2 & adpcm_e & adpcm_coder & 230 & 912 & 1493 & $(5)$ \\
\hline K3 & gsm_d & LARp_to_rp & 96 & 341 & 575 & $(1,2,1,1)$ \\
\hline K4 & gsm_d & $\begin{array}{l}\text { Short_term_ } \\
\text { synthesis_filtering }\end{array}$ & 144 & 582 & 865 & $(2)$ \\
\hline K5 & gsm_e & $\begin{array}{l}\text { Long_term_ } \\
\text { analysis_filtering }\end{array}$ & 339 & 1434 & 2061 & $(1)$ \\
\hline K6 & gsm_e & Coefficients_27_39 & 81 & 312 & 469 & $(1)$ \\
\hline K7 & gsm_e & $\begin{array}{l}\text { Short_term_ } \\
\text { analysis_filtering }\end{array}$ & 137 & 552 & 816 & $(3,2)$ \\
\hline K8 & mpeg2_d & $\begin{array}{l}\text { form_component_ } \\
\text { prediction }\end{array}$ & 960 & 4197 & 6114 & $(1)$ \\
\hline K9 & mpeg2_e & pred_comp & 925 & 4068 & 5924 & $(1)$ \\
\hline K10 & Huffman & HammingBitwise & 131 & 376 & 570 & $(1, . ., 1)^{22}$ \\
\hline
\end{tabular}

Table 1: List of kernels used. The last column shows the number of buffers inserted in each iteration of the algorithm. The Huffman circuit went through 22 iterations, with one buffer inserted in each.

is an $r \uparrow$ (see Fig. 3b), the shorter paths of the re-convergent join need only as many buffers as the number of waves of computation that could collide at the forking point. In short loops, for which the critical event after $p_{s}$ is an $a \uparrow$ event (see Fig. 3e), the waves of computation between successive iterations interfere, and these typically require more buffers.

In larger designs, re-convergent paths are dominant, and thus just a few buffers are sufficient for slack matching. In smaller designs, both types of bottlenecks may be present; moreover, fixing a re-convergent bottleneck typically gives rise to short-loop bottlenecks. Thus, more buffers are required in these cases. Acyclic designs can also exhibit bottlenecks if they are incorporated in streaming applications. For the acyclic benchmark, we injected the successive waves of inputs into the circuit, as soon as the input channels could accept them; thus we are testing its maximum capacity. Since only relative timing is measured by slack, it naturally adapts to capturing the behavior of these designs. Since we have loaded the circuit to maximum capacity, there are several waves of computation following each other in quick succession leading to the short-loop bottleneck variety. Consequently, this design required the most buffers (22), and the algorithm inserted one in each iteration of its optimization loop.

Fig. 7 shows the relative improvements in overall kernel performance and energy-delay product after applying the algorithm; a higher bar implies larger improvements. Performance refers to end-to-end latency except for the acyclic circuit (K10), for which it refers to throughput. Overall, the algorithm is capable of significantly boosting performance, over $60 \%$ in the gsm_e kernel, by inserting just a small number of buffers and running for a handful of iterations. Power increases due to the extra buffers, and also because the same amount of switching activity now occurs in a shorter time period. The overall effect, captured by the energy-delay metric, however, shows encouraging improvements. The geometric means of performance speedup and energy-delay improvements are $22 \%$ and $28 \%$ respectively for these experiments. The running time of the algorithm is less than ten seconds for all the designs listed in the table, and even lesser when no bottleneck exists. Comparatively, in previous work, the run-time to solve the LP formulation for large designs (thousands of events) take on the order of hundreds [1] or even thousands [6] of seconds in solving the optimization; in terms of the number of buffers inserted relative to the problem size, our algorithm is comparable to those reported in $[6,1]$.

\section{CONCLUSIONS}

We have described a heuristic slack matching algorithm that eliminates pipeline bottlenecks caused by mis-matched communication rates. The algorithm models system in terms of event slack and the Global Critical Path (GCP). We show that examining the communication protocol alone allows one to infer patterns, whose presence on the
GCP indicate bottlenecks due to mis-matched communication rates. Based on these observations, the algorithm identifies bottlenecks on the GCP, eliminates them by inserting pipeline buffers, updates the system state and repeats these steps until no more bottlenecks are found. In this paper, we have focused on asynchronous circuit designs, however, the proposed modeling abstraction enables portability to other design styles as well. Further, since we rely on slack and GCP metrics, the algorithm can be applied to arbitrary graph topologies and systems with choice.

Compared to the optimal solution to slack matching, which is NPcomplete, the proposed heuristic has a worst-case quadratic time complexity in the number of communication channels, and a linear time complexity, in practice. While we cannot prove whether the algorithm can converge on an optimal solution, we can bound the approximation margin for a given a solution; our experiments reveal that, in most cases, the solutions can be verified to be optimal; if not, it is within a 5\% approximation margin, in the worst case. The results also show encouraging improvements (upto 60\%) in performance and energydelay.

\section{ACKNOWLEDGMENTS}

This research has been funded by NSF ITR Scalable Molecular Electronics (contract number CCR0205523). We would like to thank Peter Beerel, Tiberiu Chelcea, Timothy Callahan and Mihai Budiu for providing invaluable insights and engaging in discussions that helped refine the ideas presented in this paper. We also thank Shivakumar Vishwanathan and Srinath Avanadhula for providing valuable feedback that vastly improved the presentation of these ideas.

\section{REFERENCES}

[1] P. Beerel, M. Davies, et al. Slack matching asynchronous designs. In ASYNC, pp. 30-39, March 2006.

[2] E. Bozorgzadeh, S. Ghiasi, et al. Optimal integer delay budgeting on directed acyclic graphs. In $D A C$, pp. 920-925, 2003.

[3] S. Burns. Performance analysis and optimization of asynchronous circuits. PhD thesis, University of Utah, 1991.

[4] S. Furber and P. Day. Four-phase micropipeline latch control circuits. IEEE TVLSI, 4-2:247-253, 1996.

[5] C. Lee, M. Potkonjak, et al. MediaBench: a tool for evaluating and synthesizing multimedia and communications systems. In MICRO, pp. 330-335, 1997.

[6] R. Lu and C.-K. Koh. Performance optimization of latency insensitive systems through buffer queue sizing of communication channels. In ICCAD, pp. 227-231, 2003.

[7] C. D. Nielsen and M. Kishinevsky. Performance analysis based on timing simulation. In DAC, pp. 70-76, 1994.

[8] P. Prakash and A. Martin. Slack matching quasi delay-insensitive circuits. In ASYNC, pp. 30-39, March 2006.

[9] I. Sutherland. Micropipelines: Turing award lecture. Communications of the ACM, 32 (6):720-738, June 1989.

[10] B. Taskin and I. Kourtev. Delay insertion method in clock skew scheduling. IEEE TCAD, 25-4:651-663, 2006.

[11] G. Venkataramani, M. Budiu, et al. C to asynchronous dataflow circuits: An end-to-end toolflow. In IWLS, June 2004.

[12] G. Venkataramani, T. Chelcea, et al. Modeling the global critical path in concurrent systems. Technical Report CMU-CS-06-144, Carnegie Mellon University, August 2006.

[13] A. Xie, S. Kim, et al. Bounding average time separations of events in stochastic timed Petri nets with choice. In ASYNC, pp. 94-107, April 1999. 